\title{
MRI T2-Weighted Imaging and Fat-Suppressed T2-Weighted Imaging Image Fusion Technology Improves Image Discriminability for the Evaluation of Anal Fistulas
}

\author{
Shi-Ting Feng, $\mathrm{PhD}^{1 *}$, Mengqi Huang, $\mathrm{BD}^{1 *}$, Zhi Dong, $\mathrm{MD}^{1}$, Ling $\mathrm{Xu}, \mathrm{MD}^{2}$, Yin $\mathrm{Li}, \mathrm{PhD}^{3}$, \\ Yingmei Jia, $\mathrm{BD}^{1}$, Huasong Cai, $\mathrm{MD}^{1}$, Bingqi Shen, $\mathrm{MD}^{1}$, Zi-Ping $\mathrm{Li}$, $\mathrm{PhD}^{1}$ \\ Departments of ${ }^{1}$ Radiology and ${ }^{3}$ Gastrointestinal Surgery, The First Affiliated Hospital of Sun Yat-Sen University, Guangzhou, China; \\ ${ }^{2}$ Faculty of Medicine and Dentistry, University of Western Australia, Perth, Australia
}

Objective: To explore whether MRI fusion technology (combined T2-weighted imaging [T2WI] and fat-suppressed T2WI [T2WI-Fs]) improves signal differences between anal fistulas and surrounding structures.

Materials and Methods: A total of 32 patients with confirmed diagnoses of anal fistula were retrospectively studied. All available T2WI and T2WI-Fs images for each patient were used to generate fusion image (T2WI-Fusion) based on the addition of gray values obtained from each pixel via an MR post-processing work station. The discriminability of fistula, perianal sphincter, and perianal fat in T2WI, T2WI-Fs, and T2WI-Fusion images was quantified with Fisher's scoring algorithm. For subjective visual image assessment by researchers, five-point scale scores were determined using a modified double-stimulus continuous qualityscale test to evaluate T2WI-rs, T2WI, enhanced axial three-dimensional-volumetric interpolated breath-hold examination (3D-VIBE), and T2WI-Fusion sequence images. The differences were subsequently compared.

Results: Mean Fisher scores for fistulas vs. sphincters obtained from T2WI-Fusion $\left(\mathrm{F}_{\text {Fusion-fistula }}=6.56\right)$ were significantly higher than those from T2WI $\left(\mathrm{F}_{\text {T2WI-fistula }}=3.35\right)(p=0.001)$. Mean Fisher scores for sphincters vs. fat from T2WI-Fusion $\left(\mathrm{F}_{\text {Fusion-sphincter }}=\right.$ 10.84) were significantly higher than those from T2WI-FS (FS Fs-sphincter $=2.57)(p=0.001)$. In human assessment, T2WI-Fusion showed the same fistula discriminability as T2WI-Fs, and better sphincter discriminability than T2WI. Overall, T2WI-Fusion showed better discriminability than T2WI, T2WI-Fs, and enhanced 3D-VIBE images.

Conclusion: T2WI and T2WI-rs fusion technology improves signal differences between anal fistulas and surrounding structures, and may facilitate better evaluation of anal fistulas and sphincters.

Keywords: Anal canal; Magnetic resonance imaging; Fusion technique; Fistula

\section{INTRODUCTION}

An anal fistula is characterized by chronic abnormal communication between the epithelialized surface of the anal canal and the perianal skin. It can be described as a narrow tunnel with its internal opening in the anal canal and its external opening in the skin near the anus (1). Detailed assessment of the anatomic relationship between the fistula and the anal sphincter complex allows surgeons to select the best surgical treatment to reduce the risk of

Received April 24, 2018; accepted after revision November 2, 2018.

This study was supported in part by grants $(81771908,81571750,81770654)$ from National Natural Science Foundation of China and by grant (2015A030313043) from the Natural Science Foundation of Guangdong Province, China.

*These authors contributed equally to this work.

Corresponding author: Zi-Ping Li, PhD, Department of Radiology, The First Affiliated Hospital of Sun Yat-Sen University, 58 Zhongshan 2nd Rd, Guangzhou, Guangdong 510080, China.

- Tel: (8620) 87755766•Fax: (8620)87615805•E-mail: liziping163@163.com

This is an 0pen Access article distributed under the terms of the Creative Commons Attribution Non-Commercial License (https:// creativecommons.org/licenses/by-nc/4.0) which permits unrestricted non-commercial use, distribution, and reproduction in any medium, provided the original work is properly cited. 
recurrence and complications such as fecal incontinence. Pre-operative confirmation of fistula complexity has been shown to both facilitate surgical planning of sphinctersaving techniques, and reduce the incidence of unidentified sepsis - the leading cause of fistula recurrence. Therefore, the role of imaging is to outline all hidden tracts, and clearly define the relationship between the fistula and the anal sphincter. Magnetic resonance imaging (MRI) provides multi-dimensional high-resolution imaging of soft tissues and is well tolerated, non-invasive, painless, and less embarrassing for the patient than other diagnostic techniques. Thus, MRI has been well accepted as the modality of choice for evaluating perianal fistulas (2).

Currently, conventional sequences for MRI scans of anal fistulas include T2-weighted imaging (T2WI), fat-suppressed T2WI (T2WI-Fs), and fat-suppressed T1-weighted imaging (T1WI-Fs). Of these scanning sequences, axial T2WI and T2WI-rs sequences are particularly important for the diagnosis of anal fistula. Perianal anatomy is best evaluated using the T2WI sequence, whereas a fistula or abscess is best evaluated using the T2WI-Fs sequence (3). The accuracy and sensitivity of MRI-based evaluations of an anal fistula are critically dependent on the acquisition of structural MRI, which are characterized by both high spatial resolution and high tissue contrast. However, conventional MRI sequences still have many shortcomings. Due to their secretionfilled nature, both anal fistulas and perianal structures demonstrate strong signals during T2WI, resulting in poor image contrast and risking misdiagnosis. Suppression of fat signals can significantly improve contrast of the fistula in T2WI-rs images but leads to poor visualization of the perianal anatomy (3). Furthermore, detection sensitivity for inactive anal fistulas that have little secretion, or for postoperative fistulas derived from scars, is lower using the T2WI-rs sequence than the T2WI sequence (4).

The utility of multiple MRI acquisition sequences for detection of soft tissue pathology using for images with different weightings has been established $(5,6)$. Combining the advantages of two or more scanning sequences into a single image potentially improves image quality, shortens image analysis time, and increases the effectiveness of diagnosis. Instead of creating new MRI scan sequences, fusion of existing high-resolution images is a valuable diagnostic tool.

In this study, we aimed to explore whether MR image fusion technology (combined T2WI and T2WI-rs) improves signal differences between anal fistulas and surrounding structures, improving visualization and evaluation of fistulas and sphincters.

\section{MATERIALS AND METHODS}

\section{Patients}

This retrospectively study was approved by our institutional ethics review board. Written informed consent was obtained from all patients in the study. About thirty-two patients with confirmed diagnoses of anal fistula, including 26 males and 6 females with a mean age of 34 years (range 12-60 years) were investigated using MRI.

\section{MRI Protocol}

All patients were scanned with a $3 T$ MRI scanner (Magnetom Avanto, version Syngo MR B17; Siemens Healthineers, Erlangen, Germany), using the body coil, with the patient in the head-first supine position and the magnetic field centered on the pubic symphysis. Imaging sequences included axial and sagittal spin echo (SE) T2WI, axial and coronal SE T2WI-Fs, axial SE T1WI, axial SE T1WI with enhanced fat suppression (T1WI-Fs), and coronal enhanced three-dimensional volumetric interpolated breathhold examination with fat suppression (E-3D-VIBE) and three-dimensional (3D) reconstruction (Table 1). Of these, axial SE T2WI and SE T2WI-rs had the same parameters (positioning line, field of view value, slice thickness, slice spacing and pixels) and are therefore represented by a single column in Table 1.

\section{MR Image Fusion}

Currently, mature image fusion techniques mainly fall into two categories: pixel-based image fusion and feature-based image fusion. The pixel-based image fusion method is also called the point-based approach, and involves calculation of either the weighted sum or the maximum or minimum of the grayscale values of the corresponding pixels of two images (5). For feature-based image fusion, operations such as feature extraction and object segmentation are performed.

Here, we applied the pixel-based image fusion approach. In digital imaging, a pixel is a physical point in a raster image, and is the smallest controllable element of a picture. A new fusion image, based on the original imaging of the axial T2WI and T2WI-rs sequences, was generated in an MR post-processing workstation (Syngo MMWP VE36, Simens Healthineers). To avoid misregistration, the fusion 
MRI T2WI Fusion Technology to evaluate Anal Fistulas

Table 1. Parameters of Sequences Used in MRI Scans of Anal Fistulas

\begin{tabular}{|c|c|c|c|c|c|c|}
\hline Parameter & T2WI & T2WI & T2WI-Fs & T2WI-Fs & T1WI & E-3D-VIBE \\
\hline Scanning plane & Axial & Sagittal & Axial & Coronal & Axial & Coronal 3D \\
\hline TR/TE (msec) & $3600 / 96$ & $3600 / 98$ & $3600 / 96$ & $4000 / 62$ & $600 / 21$ & $3.50 / 1.41$ \\
\hline FOV $(\mathrm{mm})$ & 200 & 200 & 200 & 200 & 200 & 350 \\
\hline Slice thickness (mm) & 3.0 & 3.0 & 3.0 & 3.0 & 3.0 & 1.0 \\
\hline Slice spacing $(\mathrm{mm})$ & 0.6 & 0.6 & 0.6 & 0.6 & 0.6 & 0.48 \\
\hline Pixels & 320 & 320 & 320 & 320 & 320 & 512 \\
\hline
\end{tabular}

$\mathrm{E}-3 \mathrm{D}-\mathrm{VIBE}=$ enhanced three-dimensional volumetric interpolated breath-hold examination with fat suppression, FOV $=$ field of view, TE $=$ echo time, $\mathrm{TR}=$ repetition time, T1WI $=\mathrm{T} 1$-weighted imaging, $\mathrm{T} 2 \mathrm{WI}=\mathrm{T} 2$-weighted imaging, $\mathrm{T} 2 \mathrm{WI}$ - $\mathrm{Fs}=$ fat-suppressed T2WI, 3D = three-dimensional

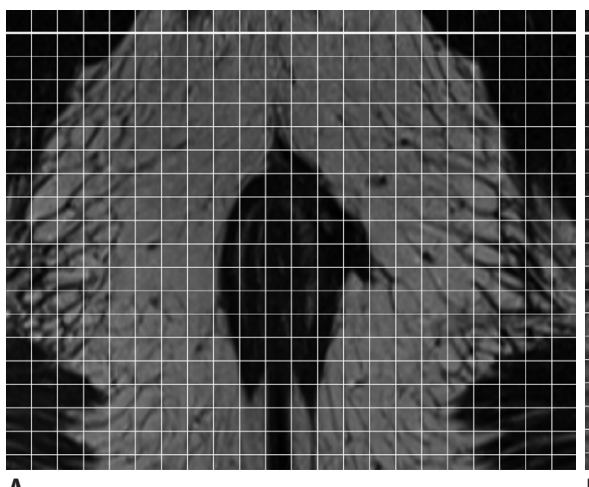

A

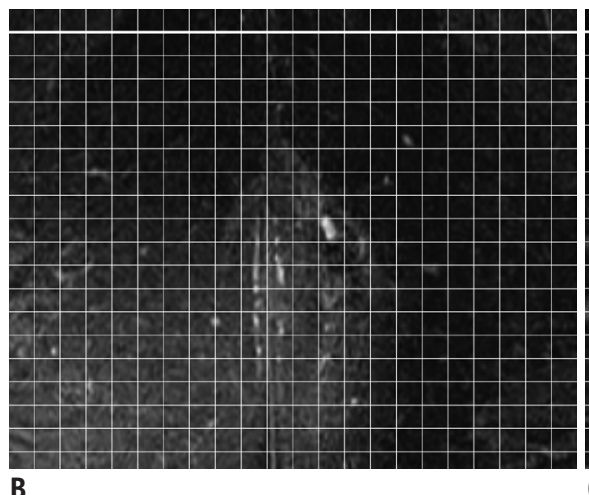

B

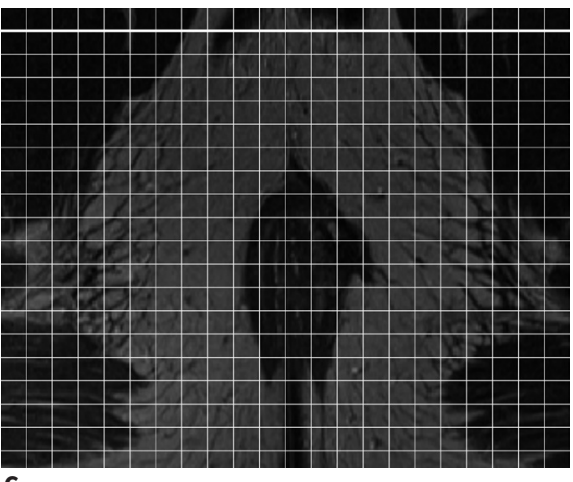

C

Fig. 1. Pixel-based magnetic resonance image fusion.

A. T2WI. B. T2WI-Fs. C. T2WI-Fusion. A and B were composed of many small pixels; sum of grayscale value in T2WI and T2WI-Fs were used in image reconstruction to generate fusion image. T2WI $=\mathrm{T} 2$-weighted imaging, $\mathrm{T} 2 \mathrm{WI}$-Fs $=$ fat-suppressed T2WI, T2WI-Fusion $=$ fusion image generated from T2WI and T2WI-Fs

images (T2WI-Fusion) was reconstructed based on the axial T2WI and T2WI-Fs sequences with the same parameters. A superposition algorithm was used and sums of the grayscale value of each corresponding pixel during both T2WI and T2WI-Fs were calculated. These data were then used to reconstruct the new fusion image of the two sequences (Fig. 1).

\section{MR Image Discriminability Evaluation}

All images were analyzed by two well-trained radiologists with 17 and 30 years of experience in abdominal diagnostic radiology, respectively. They measured the signals (means and standard deviations) of the fistula, the external sphincter, and the fat surrounding the external sphincter of three images, i.e., SE T2WI, SE T2WI-Fs and the T2WI-Fusion. The mean of the measurements taken by the two radiologists was deemed as the final measurement. The regions of interest were the maximum possible area of selection for both the fistula and the sphincter, and these selected areas were reproducible in all three images at different frequencies (Fig. 2).

The discriminability of the fusion image was evaluated according to the method of Misaki et al. (6), and tissue discriminability was calculated using the Fisher score algorithm to obtain the differences between the different tissues:

Fisher score $=(\mu \mathrm{t}-\mu \mathrm{m}) / \operatorname{sqrt}\left(\sigma \mathrm{t}^{2}+\sigma \mathrm{m}^{2}\right)$ where $\mu$ is the mean intensity of the tissue signal obtained, $\sigma$ is the standard error of the intensity of the tissue signals obtained, and $\mathrm{t}$ and $\mathrm{m}$ represent the two different tissues of interest. As the Fisher score increases, tissue discriminability increases.

As mentioned above, the $\mathrm{F}$ scores reflecting tissue discriminability between the fistula and the sphincter on T2WI ( $\left.\mathrm{F}_{\text {T2-fistula }}\right)$, and T2WI-Fusion $\left(\mathrm{F}_{\text {Fusion-fistula }}\right)$ were calculated. The $\mathrm{F}$ scores reflecting tissue discriminability between the sphincter and fat on T2WI-Fs (FFs-sphincter) and on T2WI-Fusion ( $\left.F_{\text {Fusion-sphincter }}\right)$ were also calculated.

A five-point scale was introduced for visualization assessment of image discriminability. This was determined using a modified double-stimulus continuous quality-scale (DSCQS) test to evaluate the T2WI-Fs, T2WI, axial E-3D-VIBE, and T2WI-Fusion sequence images $(7,8)$. The scale ranges from 1 to 5 . A score of 5 indicates excellent visualization, with the stricture well displayed and quickly recognizable and a noise-free image. A score of 1 indicates low image quality with severe noise, with the stricture difficult to recognize. The two observers were asked to evaluate each 


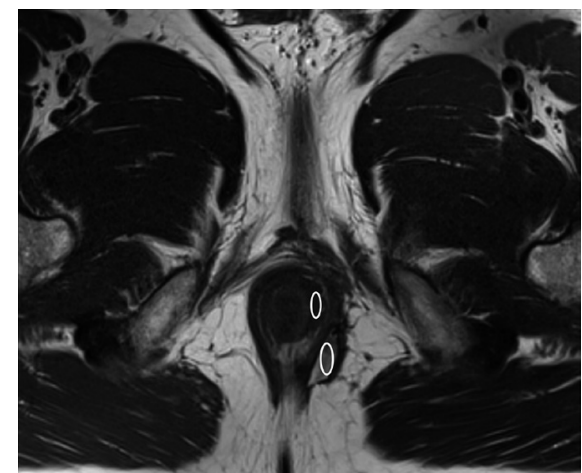

A

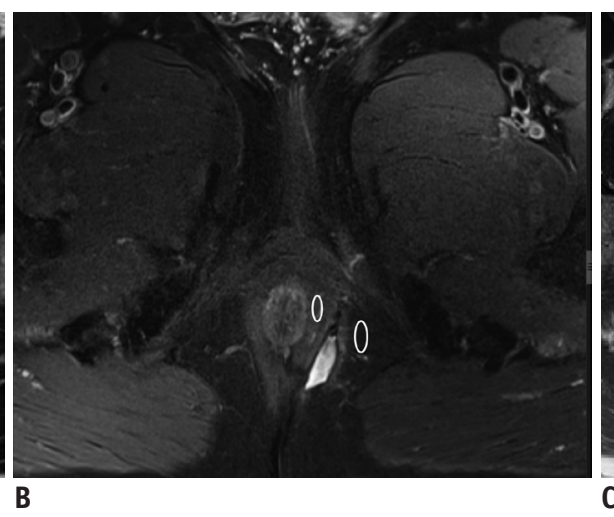

B

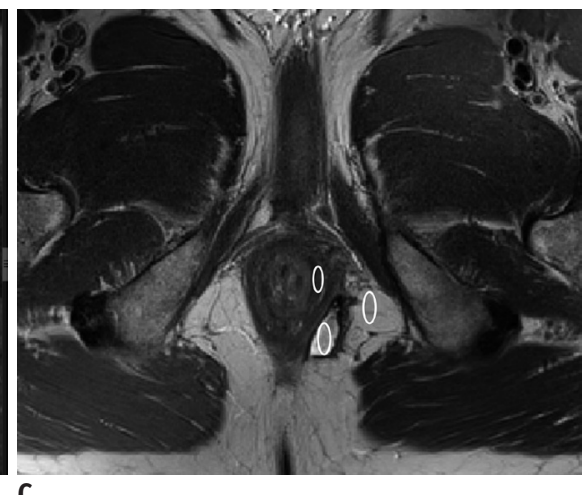

C

Fig. 2. Fisher scores for different scanning sequences.

A. T2WI. B. T2WI-Fs. C. T2WI-Fusion. Mean scores of signals for fistula, sphincter, and fat in images from three sequences were measured, and Fisher score algorithm then calculated.

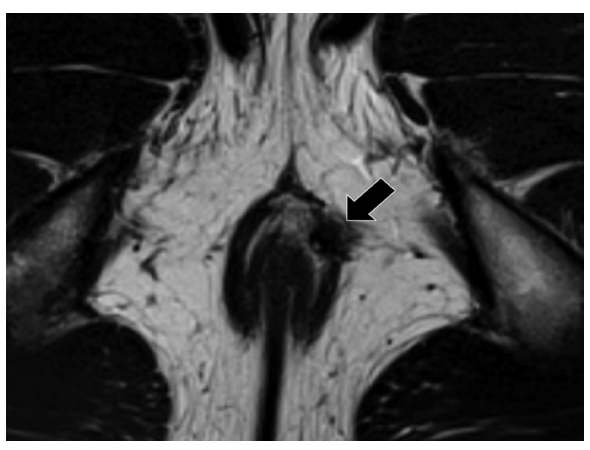

A

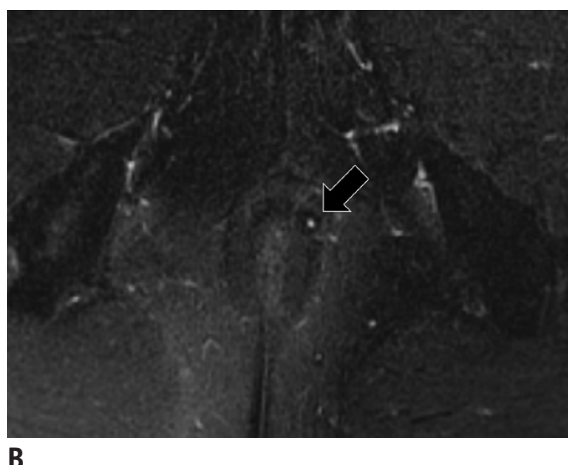

B

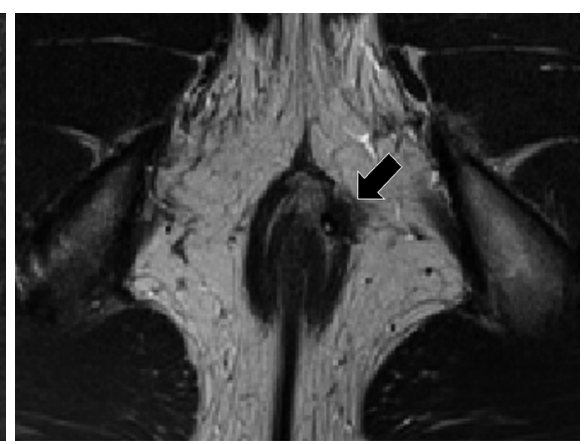

C

Fig. 3. Image of anal fistulas and perianal structures by T2WI-Fusion.

A. On T2WI, fistulas are not clearly shown (arrow). B. On T2WI-ss, fistula with high signal intensity is clearly shown (arrow), but perianal structures are not clear. C. T2WI-Fusion clearly shows fistula (arrow), and structure of internal and external sphincters.

sequence separately, and to grade the discriminability of these structures in the following separate sequence: 1 ) image discriminability of the fistula and the sphincter, separately; 2 ) image discriminability of the whole image (fistula + sphincter), scored as the sum of fistula and sphincter scores.

\section{Statistical Analysis}

The statistical software SPSS (Version 13.0; SPSS Inc., Chicago, IL, USA) was used. Fisher scores were tested for normality. Once it had been established that the four groups of Fisher scores were normally distributed, paired $t$ tests were used to compare the Fisher scores reflecting the tissue discriminability between the fistula and the sphincter, and between the sphincter and fat, obtained from the three sequences.

Paired $t$ tests were used to test for differences between image quality scores for the T2WI-Fs, T2WI, axial E-3D-VIBE and T2WI-Fusion; $p$ values less than 0.008 were considered significant according to the appropriate Bonferroni correction.

\section{RESULTS}

Fusion images (T2WI-Fusion) were successfully generated for all 32 patients. No misregistrations or obvious artifacts were found in any of the fusion images. At least one fistula was identified on each fusion image. Both fistula and sphincter structure were better displayed in the T2WI-Fusion than in the T2WI and T2WI-Fs images (Fig. 3).

A total of 160 sets of signals were generated from the T2WI, T2WI-Fs, and T2WI-Fusion images, and Fisher scores reflecting the discriminability between various tissues are shown in Table 2.

The mean Fisher score of fistula vs. sphincters for T2WI-Fusion $\left(\mathrm{F}_{\text {Fusion-fistula }}=6.56\right)$ was significantly higher than that for T2WI $\left(\mathrm{F}_{\text {T2WI-fistula }}=3.35\right)(p<0.001)$, indicating a better fistula discriminability in the former. The mean Fisher score of sphincter vs. fat for T2WI-Fusion $\left(F_{\text {Fusion-sphincter }}=10.84\right)$ was also significantly higher than that for T2WI-Fs images ( FFs- $_{\text {Fs }}$ 
sphincter $=2.57)(p<0.001)$, indicating superior sphincter discriminability on T2WI-Fusion.

In the visualization assessment of image discriminability in fistula, T2WI-rs showed the same scores as did T2WI-Fusion $(p=1.000)$, while T2WI showed the same scores as E-3D$\operatorname{VIBE}(p=0.134)$. For the visualization assessment of image discriminability in sphincter, the T2WI-Fusion demonstrated similar image discriminability as did T2WI $(p=0.031)$, and both showed better image discriminability than did T2WI-Fs $(p<0.001)$, and E-3D-VIBE $(p<0.001)$. For comparing the whole image discriminability (fistula + sphincter), T2WI-Fusion showed the best discriminability of fistula and sphincter among these four imaging sequences $(p<0.001)$ (Tables 3 , 4, Figs. 4, 5).

Table 2. Fisher Scores and Paired t Test in T2WI-Fusion, T2WI, and T2WI-rs Sequences

\begin{tabular}{lrrrc}
\hline \multicolumn{1}{c}{ Fisher Score } & Mean & SD & Variance & Paired $t$ Test $(P)$ \\
\hline F $_{\text {T2WI-fistula }}{ }^{*}$ & 3.35 & 1.70 & 2.88 & $<0.001$ \\
F Fusion-fistula $^{*}$ & 6.56 & 3.04 & 9.25 & $<0.001$ \\
F $_{\text {Fs-sphincter }}{ }^{\dagger}$ & 2.57 & 1.30 & 1.69 & $<0.001$ \\
F $_{\text {Fusion-sphincter }}{ }^{\dagger}$ & 10.84 & 4.64 & 21.54 & $<0.001$ \\
\hline
\end{tabular}

${ }^{*} \mathrm{~F}_{\text {T2WI-fistula }}$ and $\mathrm{F}_{\text {Fusion-fistula }}$ are Fisher scores reflecting tissue discriminability between fistula and sphincter on T2WI and T2WI-Fusion images, respectively, ${ }^{\dagger} \mathrm{F}_{\text {Fs-sphincter }}$ and $\mathrm{F}_{\text {Fusion-sphincter }}$ are Fisher scores reflecting tissue discriminability between sphincter and fat on T2WI-Fs and T2WI-Fusion, respectively. SD = standard deviation, T2WI-Fusion = fusion images generated from T2WI and T2WI-Fs

\section{DISCUSSION}

This study is the first application of the Fisher score and the five-point scale DSCQS test for visualization assessment of image discriminability. T2WI-Fusion demonstrated similar sphincter discriminability as T2WI, and T2WI-Fusion showed the same fistula discriminability as T2WI-rs. Among the four imaging sequences (T2WI, T2WI-Fs, T2WI-Fusion, and E-3DVIBE), T2WI-Fusion showed the best discriminability for both fistula and sphincter.

MRI has been widely recognized as the gold standard imaging modality for assessment of anal fistulas. The diagnostic values of different MRI scanning sequences for anal fistulas have been extensively investigated (9-11). Our results support previous studies concluding that the T2WI sequence yields the best representation of perianal structures $(3,4,11)$. However, due to the high signal intensities observed in fistulas and perianal fat, this sequence results in low image contrast, thereby limiting the discriminability of fistulas (3). Fistula discriminability was consequently lower in T2WI than in the T2WI-Fusion. Fusing the T2WI and T2WI-Fs images presumably enhanced relative signal intensity of the fistula, thereby showing better fistula discriminability.

The T2WI-rs sequence is relatively effective in portraying fistulas. However, the signal differences between the muscles and surrounding tissues are reduced by fat suppression, leading to poor resolution of perianal structures (3).

Table 3. Visualization Assessment of Image Discriminability

\begin{tabular}{|c|c|c|c|c|c|c|c|c|c|c|c|c|}
\hline \multirow{2}{*}{ Parameters } & \multicolumn{4}{|c|}{ Fistula } & \multicolumn{4}{|c|}{ Sphincter } & \multicolumn{4}{|c|}{ Fistula + Sphincter* } \\
\hline & T2WI-Fs & T2WI & E-3D-VIBE & T2WI-Fusion & T2WI-FS & T2WI & E-3D-VIBE & T2WI-Fusion & T2WI-Fs & T2WI & E-3D-VIBE & T2WI-Fusion \\
\hline Mean & 4.80 & 4.00 & 3.67 & 4.80 & 2.80 & 4.63 & 3.43 & 4.83 & 7.60 & 8.63 & 7.10 & 9.63 \\
\hline Median & 5 & 4 & 4 & 5 & 3 & 5 & 3 & 5 & 8 & 9 & 7 & 10 \\
\hline SD & 0.610 & 0.788 & 0.994 & 0.407 & 0.610 & 0.556 & 0.817 & 0.379 & 0.894 & 1.066 & 1.470 & 0.556 \\
\hline Min & 2 & 2 & 2 & 4 & 2 & 3 & 2 & 4 & 5 & 5 & 4 & 8 \\
\hline Max & 5 & 5 & 5 & 5 & 4 & 5 & 5 & 5 & 9 & 10 & 10 & 10 \\
\hline $25 \%$ & 5.00 & 3.75 & 3.00 & 5.00 & 2.00 & 4.00 & 3.00 & 5.00 & 7.00 & 8.00 & 6.00 & 9.00 \\
\hline $75 \%$ & 5.00 & 5.00 & 4.25 & 5.00 & 3.00 & 5.00 & 4.00 & 5.00 & 8.00 & 9.00 & 8.00 & 10.00 \\
\hline
\end{tabular}

*Sum scores of fistula and sphincter. Max = maximum, Min = minimum

Table 4. Paired $t$ Test of Visualization Assessment of Image Discriminability

\begin{tabular}{|c|c|c|c|}
\hline \multirow{2}{*}{ Sequence } & Fistula & Sphincter & Fistula + Sphincter \\
\hline & Paired $t$ Test $(P)$ & Paired $t$ Test $(P)$ & Paired $t$ Test $(P)$ \\
\hline T2WI-Fs Vs. T2WI & $<0.001$ & $<0.001$ & 0.001 \\
\hline T2WI-Fs vs. E-3D-VIBE & $<0.001$ & 0.001 & 0.053 \\
\hline T2WI-Fs vs. T2WI-Fusion & 1.000 & $<0.001$ & $<0.001$ \\
\hline T2WI vs. E-3D-VIBE & 0.134 & $<0.001$ & $<0.001$ \\
\hline T2WI vs. T2WI-Fusion & $<0.001$ & 0.031 & $<0.001$ \\
\hline E-3D-VIBE vs. T2WI-Fusion & $<0.001$ & $<0.001$ & $<0.001$ \\
\hline
\end{tabular}




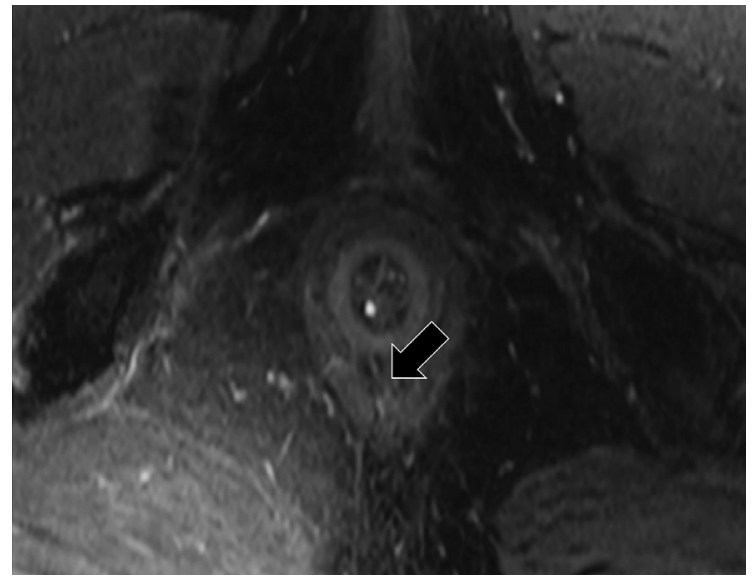

A

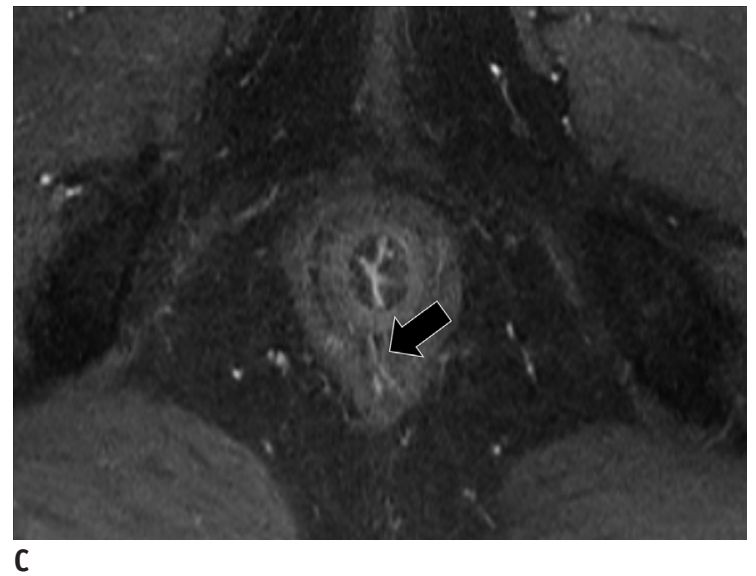

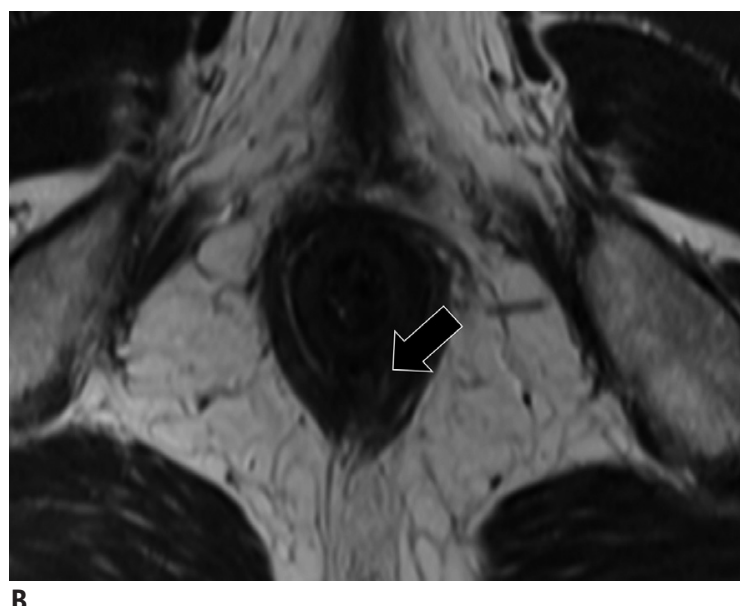

B

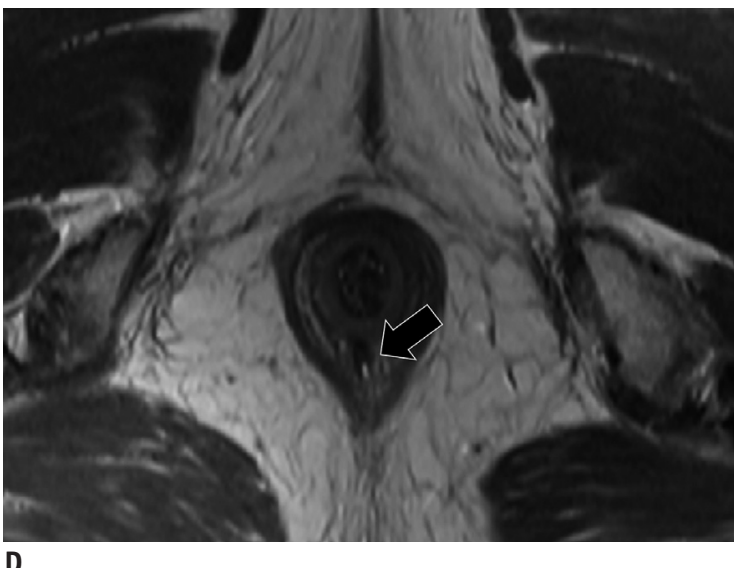

Fig. 4. Subjective image assessment with five-point scale test.

A. T2WI-Fs, shows fistula (arrow) with fistula discriminability score of 3 points and sphincter discriminability score of 2 points. B. T2WI shows fistula (arrow) with fistula discriminability score of 2 points and sphincter discriminability score of 4 points. C. E-3D-VIBE shows fistula (arrow) with fistula discriminability score of 3 points and sphincter discriminability score of 4 points. D. T2WI-Fusion shows fistula (arrow) with fistula discriminability score of 5 points and sphincter discriminability score of 5 points. E-3D-VIBE = enhanced three-dimensional volumetric interpolated breath-hold examination with fat suppression

Furthermore, compared to T2WI, the T2WI-Fs sequence limits detection of inactive anal fistulas that produce few secretions or postoperative fistulas derived from scars (4). Consequently, reconstruction of a fusion image using a pixel-based (point-based) fusion image technique combined with sum calculations of the grayscale values of the two images (T2WI and T2WI-rs) is likely to increase sphincter discriminability while retaining good fistula discriminability.

Fistula representation in the resulting fusion images showed remarkably high signals, and was superior to that obtained with T2WI. Moreover, healing fistulas can be better visualized using T2WI-Fusion, which shows clear fiber rings with lower signal intensities surrounding most parts of the fistulas. These may be missed using T2WI-rs sequences. In addition, fusion images depicted the perianal structures better than did T2WI-Fs. This improvement was due to the addition of more signals to the original fistula signal when the pixel superimposition technique was used to generate the fusion images. Fistula signals were higher in T2WIFs than in T2WI, and this localized signal enhancement substantially increased the contrast between fistulas and the surrounding muscle and fat, which exhibited unchanged signal intensity. Thus, fistula resolution improved significantly in fused images, which also retained the advantages of T2WI for displaying perianal structures. Furthermore, fibrosis is a common finding in evaluations of the peripheries of anal fistulas, and is observed at low signal intensities in T2WI and T2WI-rs and still lower intensities in fusions of these two image types. This fusion technique thus greatly facilitates clear portrayal of fistulas. In this study, T2WI-Fusion also demonstrated better fistula and sphincter discriminability relative to that of E-3D-VIBE 

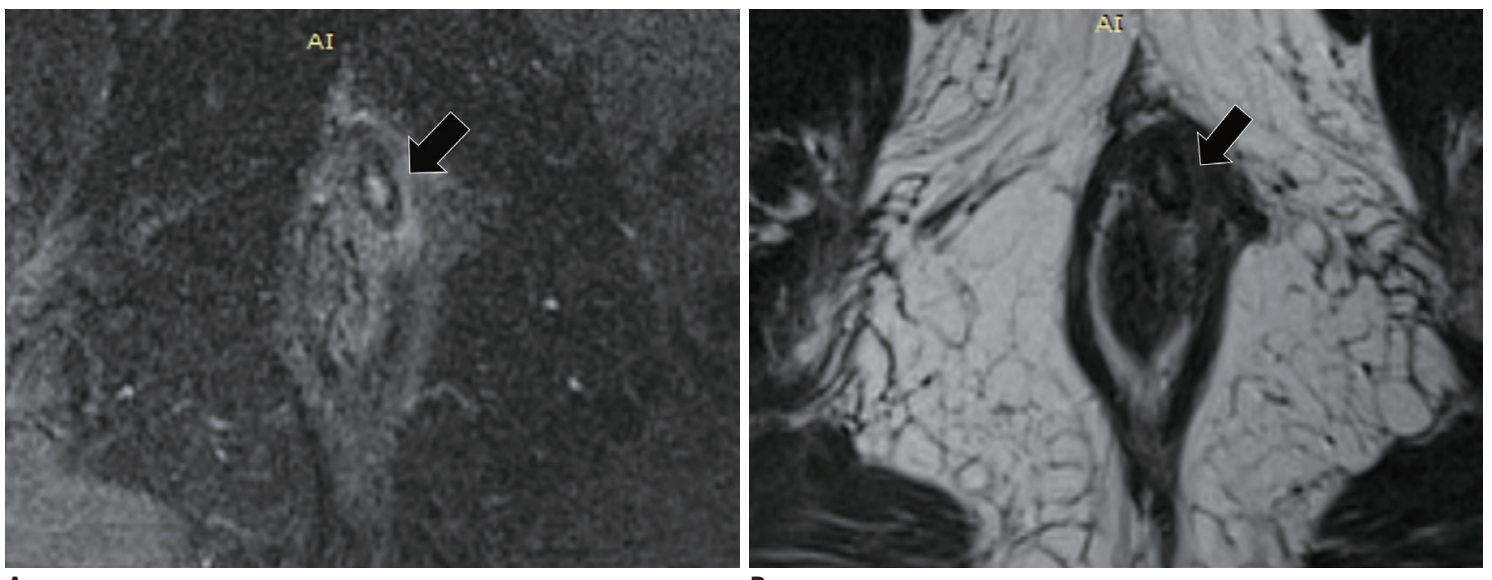

A

B
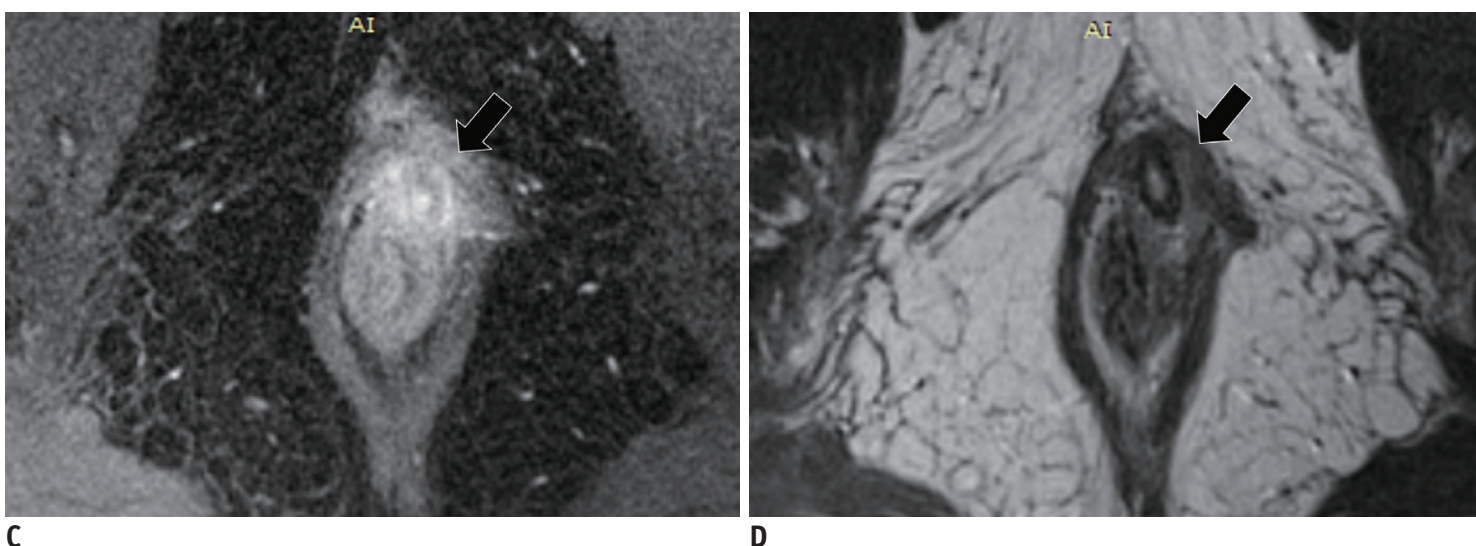

Fig 5. Image discriminability of intersphincter anal fistula in different MRI sequences.

A. T2WI-Fs. B. T2WI. C. E-3D-VIBE. D. T2WI-Fusion. Fistula (arrow) was displayed in 4 images, T2WI-Fusion showed best discriminability (fistula + sphincter) among these four sequences, and fistula (arrow) was displayed well on T2WI-Fusion and T2WI-rs, while it was worse displayed in E-3D-VIBE images than on T2WI-fusion image. Image discriminability of sphincter on T2WI-Fusion was as good as that of T2WI, but better than that of T2WI-Fs.

images. This may be explained by the combined advantages of high fistula discriminability in T2WI-Fs and high sphincter discriminability in T2WI, both evident in T2WI-Fusion.

Enhanced T1WI both displays the course of perianal fistulas and reflects their inflammatory activity well (3).

E-3D-VIBE is a good image sequence for fistula detection and assessment. However, the assessment of fistula delineation and relationship with the sphincter is influenced by enhancement of the fistula. Particularly in active disease, image enhancement of both the fistula and the sphincter results in decreased signal differences between the two. Ziech el al. (10) also reported that dynamic contrast-enhanced MRI correlates with disease activity and may have utility as an indicator of therapy effect. However, the role of T2WI-Fusion in reflecting inflammatory activity of the anal fistula remains uncertain. In such instances, use of contrast-enhanced T1WI may still be an essential component for evaluating anal fistulas.
Substantial efforts have been made to improve the diagnosis of anal fistulas using MR, including the development of new scanning sequences such as diffusion-weighted imaging, liver acquisition with volume acceleration flexible, and other methods (12-16). Unlike previous studies, this study indicates that MR fusion techniques can significantly improve the diagnosis of anal fistulas through manipulation of existing conventional scanning sequences without prolonging MR scanning time. This time-efficient technique can significantly improve both image quality and MR diagnostic value for anal fistulas using simple, applicable post-imaging data reconfiguration algorithms. In addition, our comparisons of image quality were free of confounding factors such as surgery, pathology, and the type of image analyzer because we used objective measurements to assess the quality of fusion images directly.

Image fusion techniques occupy an important position in 
the field of medical diagnostic imaging. Currently, the most widely used and successful image fusion technique is the combination of positron emission tomography (PET) and computed tomography (CT) imaging to integrate functional and anatomical data, greatly improving diagnostic accuracy (17). Developments in image processing technology are increasingly enabling researchers to generate fusion images that improve the diagnosis of lesions. The source images are often acquired using methods that require sophisticated equipment and software, such as PET and MRI, ultrasound imaging and MRI, and MRI and CT imaging (18-20). However, fusion images from MRI have been rarely reported, although Misaki et al. (6) generated a fusion image from T1WI and T2WI using MR brain scans, and adopted an algorithm to produce fusion images that showed better consistency and contrast between the gray and white matter of the brain. Pixel-based MRI fusion has the advantages of requiring neither complex mathematical algorithms nor increased scan time; moreover, its operation is simple and will play an increasingly important role in the imaging diagnosis of disease.

This study proved that T2WI-Fusion show great advantages in displaying anal fistulas and sphincters. We believe that the benefits of combining multiple contrasts could be further enhanced by optimization of imaging parameters. Our study supports the use of fusion images during the delineation of the anal fistula tract, especially in healed cases. However, in cases with active inflammatory processes or extensive abscess formation, the discriminability of fusion images may not be helpful, because acute inflammation processes or abscesses obliterate signal intensity of the desired anatomical features.

The main limitation of our study is the lack of operative findings to use as the gold standard to compare T2WI-Fusion and T2WI or T2WI-Fs, and thereby evaluate the diagnostic effectiveness of image fusion for anal fistulas. Secondly, this is a retrospective study; therefore, patient selection bias could not be avoided. Lastly, the utility of T2WIFusion for anal fistula cases with active inflammation should be further evaluated by collection of images from more patients.

In summary, T2WI and T2WI-rs fusion technology improve signal differences between anal fistulas and surrounding structures, and may thereby improve evaluation of anal fistulas and sphincters. As an alternative to the generation of new MRI scan sequences, this MRI fusion technique has the advantages of being time-efficient and operator- convenient. It is highly feasible to apply this technique for future evaluations of anal fistulas and sphincters.

\section{Conflicts of Interest}

The authors have no financial conflicts of interest.

ORCID
Zi-Ping Li
https://orcid.org/0000-0003-0041-4062
Shi-Ting Feng
https://orcid.org/0000-0002-0869-7290
Mengqi Huang
https://orcid.org/0000-0002-6976-9964

\section{REFERENCES}

1. Liang C, Lu Y, Zhao B, Du Y, Wang C, Jiang W. Imaging of anal fistulas: comparison of computed tomographic fistulography and magnetic resonance imaging. Korean $\mathrm{J}$ Radiol 2014;15:712-723

2. Baskan 0, Koplay M, Sivri M, Erol C. Our experience with MR imaging of perianal fistulas. Pol J Radiol 2014;79:490-497

3. de Miguel Criado J, del Salto LG, Rivas PF, del Hoyo LF, Velasco LG, de las Vacas MI, et al. MR imaging evaluation of perianal fistulas: spectrum of imaging features. Radiographics 2012;32:175-194

4. Lo Re G, Tudisca C, Vernuccio F, Picone D, Cappello M, Agnello $F$, et al. MR imaging of perianal fistulas in Crohn's disease: sensitivity and specificity of STIR sequences. Radiol Med 2016;121:243-251

5. Inoue HK, Nakajima A, Sato H, Noda SE, Saitoh J, Suzuki $\mathrm{Y}$. Image fusion for radiosurgery, neurosurgery and hypofractionated radiotherapy. Cureus 2015;7:e252

6. Misaki M, Savitz J, Zotev V, Phillips R, Yuan H, Young KD, et al. Contrast enhancement by combining T1- and T2-weighted structural brain MR Images. Magn Reson Med 2015;74:16091620

7. Huo D, Salem KA, Jiang Y, Wilson DL. Optimization of spiral MRI using a perceptual difference model. Int J Biomed Imaging 2006;2006:35290

8. ITU Radiocommunication Assembly. Recommendation ITU-R BT.500-11. Methodology for the subjective assessment of the quality of television pictures [updated 2002]. International Telecommunication Union. Available at: https://www.itu.int/ dms_pubrec/itu-r/rec/bt/R-REC-BT.500-11-200206-S!!PDF-E. pdf. Accessed June, 2002

9. Singh K, Singh N, Thukral C, Singh KP, Bhalla V. Magnetic resonance imaging (MRI) evaluation of perianal fistulae with surgical correlation. J Clin Diagn Res 2014;8:RC01-RC04

10. Ziech ML, Lavini C, Bipat S, Ponsioen CY, Spijkerboer AM, Stokkers PC, et al. Dynamic contrast-enhanced MRI in determining disease activity in perianal fistulizing 
Crohn disease: a pilot study. AJR Am J Roentgenol 2013;200:W170-W177

11. Hu D, Wang C, Langer M. [Comparison of MR-sequence in the diagnosis of anal fistula--the clinical value of 3D flash]. J Huazhong Univ Sci Technolog Med Sci 2002;22:241-245

12. Yoshizako T, Kitagaki H. A pictorial review of the impact of adding diffusion-weighted MR imaging to other MR sequences for assessment of anal fistulae. Jpn J Radiol 2013;31:371-376

13. Yoshizako T, Wada A, Takahara T, Kwee TC, Nakamura M, Uchida K, et al. Diffusion-weighted MRI for evaluating perianal fistula activity: feasibility study. Eur J Radiol 2012;81:2049-2053

14. Cavusoglu M, Duran S, Sözmen Cılız D, Tufan G, Hatipoglu Çetin HG, Ozsoy A, et al. Added value of diffusion-weighted magnetic resonance imaging for the diagnosis of perianal fistula. Diagn Interv Imaging 2017;98:401-408

15. Szyszko TA, Bush J, Gishen P, Sellu D, Desouza NM. Endoanal magnetic resonance imaging of fistula-in-ano: a comparison of STIR with gadolinium-enhanced techniques. Acta Radiol
2005;46:3-8

16. Xiaoming $L$, Rongrong $G$, Shengpan J. The study on the diagnostic value with LAVA-FLEX sequence for diagnosing anal fistula. Chinese Journal of CT and MRI 2015;(6):80-83

17. Antonica F, Asabella AN, Ferrari C, Rubini D, Notaristefano A, Nicoletti A, et al. Useful diagnostic biometabolic data obtained by PET/CT and MR fusion imaging using open source software. Hell J Nucl Med 2014;17 Suppl 1:50-55

18. Loeffelbein DJ, Souvatzoglou M, Wankerl V, Dinges J, Ritschl LM, Mücke T, et al. Diagnostic value of retrospective PET-MRI fusion in head-and-neck cancer. BMC Cancer 2014;14:846

19. Peach MS, Trifiletti DM, Libby B. Systematic review of focal prostate brachytherapy and the future implementation of image-guided prostate HDR brachytherapy using MRultrasound fusion. Prostate Cancer 2016;2016:4754031

20. Barnaure I, Pollak P, Momjian S, Horvath J, Lovblad KO, Boëx C, et al. Evaluation of electrode position in deep brain stimulation by image fusion (MRI and CT). Neuroradiology 2015;57:903-908 